\title{
Role of Luteal Phase Endometrial Injury in Outcome of In Vitro Fertilization
}

\author{
Isha Kriplani ${ }^{1}$, Swati Garg ${ }^{2}$, Priyanka Goel ${ }^{3}$, Manisha Chowdhary ${ }^{4}$
}

\begin{abstract}
Introduction: The development of receptive endometrium is necessary for successful implantation, which is now considered a rate-limiting step for the success of in vitro fertilization (IVF). Lately, scratching of endometrium before embryo transfer (ET) is being investigated as a possible technique to increase implantation rate. By improving endometrial receptivity, the endometrial injury might increase implantation and clinical pregnancy rates.

Materials and methods: A total of 100 patients, who met the inclusion criteria, were randomized into two groups, group I (study group) in which endometrial scratching was done before ET, and group II (control group) in which endometrial scratching was not done. All the patients had previous history of implantation failure at one or more occasions.

Results: In group I, 19/35 patients who underwent IVF conceived whereas in group II, $9 / 30$ patients conceived. The conception rate in intervention group was higher than the control group for patients who underwent conventional IVF and this difference was found to be statistically significant, $p$ value 0.049 . In group I, 29 (58.0\%) babies were born to 50 women who underwent intervention and in group II, 15 babies were born. This difference in carry-home baby rate was statistically significant $(p=0.005)$.

Conclusion: The benefit of luteal phase endometrial injury cannot be refuted and larger multicenter randomized controlled trials are required for conclusive evidence.

Keywords: Endometrium, Implantation failure, IVF, Pregnancy.

Journal of Mahatma Gandhi University of Medical Sciences \& Technology (2020): 10.5005/.jp-journals-10057-0132
\end{abstract}

\section{INTRODUCTION}

Infertility is "a disease of the reproductive system defined by the failure to achieve a clinical pregnancy after 12 months or more of regular unprotected sexual intercourse". ${ }^{1}$ WHO estimates the prevalence of primary infertility in India to be between 3.9 and $16.8 \%{ }^{1}$

Evidence indicating that about $75 \%$ of human pregnancies fail after conception implicates early embryopathy or implantation failure as the possible causes of unexplained infertility. ${ }^{2}$ Interactions between the endometrium and the embryo as well as endometrial receptivity are considered as two strong factors affecting the outcome of implantation. $^{3}$

Lately, scratching of endometrium before embryo transfer (ET) is being investigated as a possible technique to increase implantation rate. There are three possible mechanisms. ${ }^{4}$ According to the first mechanism, local injury to proliferative phase endometrium might induce the decidualization of endometrium and increase its implantation rate, the second one says that local injury to the endometrium might provoke the wound healing, involving a massive secretion of different cytokines and growth factors, including leukemia inhibitory factor (LIF) and interferon-11 (IF-11) which are beneficial for embryo implantation, ${ }^{5,6}$ thirdly "The Backward development hypothesis" states that local injury to the endometrium during the controlled ovarian hyperstimulation cycle might result in a "lag" in the development of endometrium because of the wound, so that development of endometrium would be more equivalent with embryo development and also increase its receptivity.

This study was planned in view of the above-stated hypothesis thus enhancing the probability of implantation and
${ }^{1-3}$ Department of Obstetrics and Gynaecology, Mahatma Gandhi Medical College and Hospital, Jaipur, Rajasthan, India

${ }^{4}$ Department of Reproductive Medicine, Mahatma Gandhi Medical College and Hospital, Jaipur, Rajasthan, India

Corresponding Author: Swati Garg, Department of Obstetrics and Gynaecology, Mahatma Gandhi Medical College and Hospital, Jaipur, Rajasthan, India, Phone: +919414048000, e-mail: drswati_garg@ hotmail.com

How to cite this article: Kriplani I, Garg S, Goel P, et al. Role of Luteal Phase Endometrial Injury in Outcome of In Vitro Fertilization. J Mahatma Gandhi Univ Med Sci Tech 2020;5(2):47-49.

Source of support: Nil

Conflict of interest: None

successful outcome in patients undergoing in vitro fertilization (IVF)-ET cycles.

\section{Materials and Methods}

The present study was conducted at the ART center of Mahatma Gandhi Medical College and Hospital, Jaipur. Ethical approval for the study was obtained from the Institute Ethics Committee. A total of 100 patients, who met the inclusion criteria, were randomized into two groups, after taking the informed consent, group I (study group) in which endometrial scratching was done in the secretory phase (D20-24) in the cycle preceding the ET cycle and group II (control group) in which endometrial scratching was not done. All the patients had a previous history of implantation failure on one or more occasions. 


\section{Inclusion and Exclusion Criteria}

\section{Inclusion Criteria}

- Age $<35$ years.

- Patients with at least two previous failed IVF-ET/intracytoplasmic sperm insemination (ICSI) cycles.

- Good responders in the previous IVF cycle*.

- No uterine manipulation within the last 3 months (e.g., myomectomy).

\section{Exclusion Criteria}

- Women with a history of septal resection, adhesiolysis, Asherman's syndrome, and abnormal uterine cavity.

- History of endometrial tuberculosis in the past.

- Women with possible medical causes for failure of implantation, e.g., diabetes mellitus, hypertension, and autoimmune diseases.

- Presence of sonographically detected hydrosalpinx or endometriosis.

*Good responders are defined as the patients who develop at least four good-quality embryos (grade I and II of Veeck's grading) in the previous IVF cycles.

\section{Study Method}

All patients were evaluated with baseline anti mullerian hormone (AMH) (day 3), follicle stimulating factor (FSF), and a day 12 ultrasound for measuring endometrial thickness, quality, and blood flow. Records of previous stimulation protocols and embryology details were reviewed.

After obtaining informed consent, the patients in the intervention group underwent endometrial scraping, with a biopsy catheter (Pipelle), on day 20-24 of the non-transfer cycle on outpatient basis.

Each woman recruited in the study underwent the same controlled ovarian hyperstimulation protocol $(\mathrm{COH})$ that she had undergone in the previous IVF cycles. Oocyte retrieval followed by insemination/ICSI and ET was done.

Serum beta-human chorionic gonadotropin (hCG) was checked in all women on day 15 after the transfer. Those with positive beta-hCG were confirmed for clinical pregnancy by sonography 2 weeks after the beta-hCG report. Clinical pregnancy was defined as the presence of an intrauterine gestational sac with embryonic cardiac activity observed by vaginal ultrasound. After the successful implantation, follow-up of pregnancy was done till term.

\section{Statistical Analysis}

All data analysis was carried out using the statistical package SPSS IBM version 19.0. After testing for normality assumptions, using appropriate statistics, mean values were compared between two groups using Student's " $t$ " independent test. Frequency distributions of categorical variables were compared using Chisquare/Fisher's exact test as appropriate. For all statistical tests, the probability of $p<0.05$ was considered for statistical significance.

\section{Results}

According to the results obtained, the mean age was $31.48 \pm$ 3.23 and $30.90 \pm 3.32$ years in groups I and II, respectively, with $p$ value $>0.05$, the difference being not significant. Sixty-six percent of patients in group I and $64 \%$ in group II had primary infertility. The duration of infertility was $7.86 \pm 4.05$ in both groups and the
Table 1: Comparison between various parameters

\begin{tabular}{|c|c|c|c|}
\hline & $\begin{array}{l}\text { Group I, mean } \\
\pm S D\end{array}$ & $\begin{array}{l}\text { Group II, mean } \\
\pm S D\end{array}$ & $p$ value \\
\hline Age (years) & $31.48 \pm 3.23$ & $30.90 \pm 3.32$ & 0.378 \\
\hline Type of infertility & & & 0.834 \\
\hline Primary & $33(66 \%)$ & $32(64 \%)$ & \\
\hline Secondary & $17(34)$ & $18(36)$ & \\
\hline $\begin{array}{l}\text { Duration of } \\
\text { infertility (years) }\end{array}$ & $7.86 \pm 4.00$ & $7.86 \pm 4.05$ & 1.000 \\
\hline $\begin{array}{l}\text { Attempts of IVF/ } \\
\text { ICSI }\end{array}$ & $2.48 \pm 0.79$ & $2.36 \pm 0.60$ & 0.393 \\
\hline
\end{tabular}

Table 2: Outcome measures

\begin{tabular}{llll}
\hline & Group I & Group II & p value \\
\hline $\begin{array}{l}\text { Conception rate } \\
\text { Clinical pregnancy }\end{array}$ & $26 / 50(52 \%)$ & $18 / 50(36 \%)$ & 0.107 \\
rate & & $15(30 \%)$ & 0.271 \\
Abortion rate & $4 / 26(15.3 \%)$ & $4 / 18(22.2 \%)$ & 0.697 \\
$\begin{array}{l}\text { Live birth rate } \\
\begin{array}{l}\text { Carry-home baby } \\
\text { rate }\end{array}\end{array}$ & $22 / 50(44.0 \%)$ & $13 / 50(26.0 \%)$ & 0.059 \\
& & & 0.005 \\
\hline
\end{tabular}

mean attempts of IVF/ICSI were also similar with $p$ value being not significant between both the groups as shown in Table 1. The various outcomes of pregnancy were analyzed in the study and have been shown in Table 2. There was an increase in the clinical pregnancy rate and decrease in abortion rate in the intervention group (44 vs $30 \%$ and 15.3 vs $22.2 \%$, respectively) which might be due to increased implantation rate due to endometrial scratching. Similarly, the carry home baby rate was also higher in the study group as compared to the control group (58 vs 30\%), the difference being statistically significant.

\section{Discussion}

The endometrial injury appears to induce changes in the endometrium that might facilitate implantation of the embryo in women undergoing ART. Studies in the past have supported this view.

In the present study, the mean age of women was $31.48 \pm 3.23$ years in group I and $30.90 \pm 3.32$ years in group II.

In the study by İnal et al. 2012, ${ }^{7}$ the mean age of the control group was $30.8 \pm 4.5$ years and for the study group it was $29.6 \pm$ 3.8 years. Similarly, in the randomized controlled trial (RCT) done by Parsanezhad et al. 2013, ${ }^{8}$ study group had mean age of $30.0 \pm$ 3 years and control group had mean age of $30.32 \pm 2$ years. The mean age of our study was comparable to all of the above studies.

The type of infertility was also comparable between the two groups but this parameter was not compared in any other study.

In the present study, the mean duration of infertility in group I was $7.86 \pm 4.00$ years and in group II was $7.86 \pm 4.05$ years. In the study conducted by Raziel et al. $2007,{ }^{9}$ the mean duration of infertility in intervention group was $4.9 \pm 2.9$ years and in control group was $5.5 \pm 3.0$ years which was less than that in our study. In the present study, the mean attempts of IVF/ICSI in group I was $2.48 \pm 0.79$ whereas that in group II was $2.36 \pm 0.60$. Raziel et al. in $2007^{9}$ found that the no of previous ICSI cycles was $7.0 \pm 1.9$ in intervention group and $5.7 \pm 1.0$ in control group, $p$ value $<0.003$; hence, there was a statistically significant difference between the 
two groups in their study. The mean attempts in this study were higher than that in the present study.

In our study, 26/50 patients conceived making the conception rate $52 \%$ in the study group and $18 / 50$ patients conceived, conception rate being $36 \%$ in the control group. This was not studied in any other study.

$15.4 \%$ of patients aborted in the intervention group and $22.2 \%$ in the control group in the present study but the difference was not significant statistically. Similarly, Parsanezhad et al. in $2013^{8}$ also did not find any significant difference between the two groups regarding the abortion rate ( 17.64 vs $14.28 \% ; p=0.701)$. Kumbak et al. in $2014^{10}$ reported that the miscarriage rate was higher in the study group as compared to the control group, but this difference was not statistically significant.

In the present study, in group I, $44 \%$ and $26 \%$ in group II had live birth but it was not statistically significant.

In the study done by Parsanezhad et al. in $2013,{ }^{8}$ the ongoing pregnancy rate was significantly higher in the endometrial injury group compared to the control group (14.9 vs $5.8 \%$; $p=0.03$ ). Kumbak et al. in $2014^{10}$ also found a higher ongoing or live birth rate in the study group as compared to the control group, but it was also not statistically significant (54 vs $38 \% ; p>0.05$ ).

However, Karimzade et al. in $2010^{11}$ conducted RCT and reported statistically significant lower ongoing pregnancy rate in the study group as compared to the control group ( 9.6 vs $29.1 \%$; $p=0.004)$. Baum et al. in $2012^{12}$ also reported lower live birth rate in study group as compared to control group (0 vs $25 \%$; $p=0.1$ ).

\section{Conclusion}

Implantation rate increases after endometrial scratching in the nontransfer cycle in patients with previous failed IVF-ET. Endometrial scratching initiates changes within the endometrium, the immune system, and gene expression, all leading to improved receptivity and a favorable environment for implantation. Injury in the preceding cycle is more effective as all these events require time. Intervention, when done near to ET, can negatively affect implantation rate by potentially disturbing the endometrium receptivity. However, large multicenter randomized studies are needed to investigate the role of endometrial injury and pregnancy outcomes in the unexplained recurrent implantation failure (RIF), as regarding the timing and whether repeating the procedure in women who failed to conceive after undergoing endometrial scratching once may be beneficial or not, and to explore the mechanism of increased receptivity to implantation of the injured endometrium involving molecular studies.

\section{References}

1. WHO|Global prevalence of infertility, infecundity and childlessness [Internet]. WHO. [cited 2013 Oct 20]. Available from: http://www.who. int/reproductivehealth/topics/infertility/burden/en/index.html.

2. Macklon NS, Geraedts JPM, Fauser BCJM. Conception to ongoing pregnancy: the "black box" of early pregnancy loss. Hum Reprod Update 2002;8(4):333-343. DOI: 10.1093/humupd/8.4.333.

3. Karimzadeh MA, AyaziRozbahani M, Tabibnejad N. Endometrial local injury improves the pregnancy rate among recurrent implantation failure patients undergoing in vitro fertilisation/intra cytoplasmic sperm injection: a randomised clinical trial. Aust $\mathrm{N} Z \mathrm{~J}$ Obstet Gynaecol 2009;49(6):677-680. DOI: 10.1111/j.1479-828X.2009. 01076.x.

4. Zhou L, Li R, Wang R, et al. Local injury to the endometrium in controlled ovarian hyperstimulation cycles improves implantation rates. Fertil Steril 2008;89(5):1166-1176. DOI: 10.1016/j.fertnstert.2007. 05.064.

5. Sherer DM, Abulafia O. Angiogenesis during implantation, and placental and early embryonic development. Placenta 2001;22(1):113. DOI: $10.1053 /$ plac. 2000.0588.

6. Akita $\mathrm{S}$, Ishihara $\mathrm{H}$, Mohammad Abdur R, et al. Leukemia inhibitory factor gene improves skin allograft survival in the mouse model. Transplantation 2000;70(7):1026-1031. DOI: 10.1097/00007890200010150-00007.

7. Inal ZHÖ, Görkemli H, Inal HA. The effect of local injury to the endometrium for implantation and pregnancy rates in ICSI-ET cycles. Eur J Gen Med 2012;9(4):223-229.

8. Parsanezhad ME, Dadras N, Maharlouei N, et al. Pregnancy rate after endometrial injury in couples with unexplained infertility: a randomized clinical trial. Iran J Reprod Med 2013;11(11): 869-874.

9. Raziel A, Schachter M, Strassburger D, et al. Favorable influence of local injury to the endometrium in intracytoplasmic sperm injection patients with high-order implantation failure. Fertil Steril 2007;87(1):198-201. DOI: 10.1016/j.fertnstert.2006.05.062.

10. Kumbak B, Sahin L, Ozkan S, et al. Impact of luteal phase hysteroscopy and concurrent endometrial biopsy on subsequent IVF cycle outcome. Arch Gynecol Obstet 2014;290(2):369-374. DOI: 10.1007/ s00404-014-3211-y.

11. Karimzade MA, Oskouian $\mathrm{H}$, Ahmadi S, et al. Local injury to the endometrium on the day of oocyte retrieval has a negative impact on implantation in assisted reproductive cycles: a randomized controlled trial. Arch Gynecol Obstet 2010;281(3):499-503. DOI: 10.1007/s00404009-1166-1.

12. Baum M, Yerushalmi GM, Maman E, et al. Does local injury to the endometrium before IVF cycle really affect treatment outcome? results of a randomized placebo controlled trial. Gynecol Endocrinol Off J Int Soc Gynecol Endocrinol 2012;28(12):933-936. DOI: 10.3109/09513590.2011.650750 